\title{
miR-221-3p promotes the cell growth of non-small cell lung cancer by targeting p27
}

\author{
GUOQING YIN ${ }^{1}$, BO ZHANG ${ }^{2}$ and $\mathrm{JIA} \mathrm{LI}^{3}$ \\ ${ }^{1}$ Department of Oncology, Xianyang Hospital, Yan'an University, Xianyang, Shaanxi 712000; \\ ${ }^{2}$ Radiation Department, People's Hospital of Ankang City, Ankang, Shaanxi 725000; \\ ${ }^{3}$ Department of Respiratory Medicine, Longnan Hospital, Daqing, Heilongjiang 163453, P.R. China
}

Received July 28, 2018; Accepted February 21, 2019

DOI: $10.3892 / \mathrm{mmr} .2019 .10291$

\begin{abstract}
Emerging evidence suggests the critical function of microRNAs in regulating the growth of cancer cells. In the present study, it was demonstrated that miR-221-3p was overexpressed in non-small cell lung cancer (NSCLC) tissues and cell lines compared with that noted in the normal controls. Downregulation of miR-221-3p suppressed the proliferation, colony formation and invasion of NSCLC cells. To further understand the molecular mechanisms underlying the potential oncogenic function of miR-221-3p in NSCLC, the downstream targets of miR-221-3p were predicted using bioinformatic databases. The prediction suggested the cell cycle regulator $\mathrm{p} 27$ as one of the targets of miR-221-3p. Molecular experiments showed that miR-221-3p was able to bind with the 3'-untranslated region (UTR) of p27 and decreased the expression of p27 in NSCLC cells. Consistent with the suppressive role of $\mathrm{p} 27$ in controlling cell cycle progression, overexpression of miR-221-3p decreased the expression of p27 and promoted cell cycle progression from G1 to $\mathrm{S}$ phase. Collectively, our findings identified miR-221-3p as a novel regulator of NSCLC cell growth via modulating the expression of $\mathrm{p} 27$.
\end{abstract}

\section{Introduction}

Lung cancer is the leading cause of cancer-associated mortality worldwide (1). The prevention and treatment of lung cancer have drawn wide attention and have been a focus in clinical practice. Non-small cell lung cancer (NSCLC), which initiates from non-small cells of the lung, accounts for approximately $85 \%$ of all lung cancer cases with a high mortality rate (2). In the past decade, surgical resection combined with chemotherapy has greatly improved the prognosis of lung cancer patients; however, due to the fact

Correspondence to: $\mathrm{Dr} \mathrm{Jia} \mathrm{Li}$, Department of Respiratory Medicine, Longnan Hospital, 35 Patriotic Road, Ranghulu, Daqing, Heilongjiang 163453, P.R. China

E-mail: surgical_zx@126.com

Key words: miR-221-3p, p27, NSCLC, cell cycle that most patients are diagnosed with middle or late stage of the disease, the 5-year survival rate of NSCLC patients still remains poor. Therefore, exploration of the underlying molecular mechanisms which regulate tumor growth is important in searching for effective targets that may benefit the treatment of NSCLC.

MicroRNAs (miRNAs) are a class of non-coding regulatory RNAs with a length of 20-24 nucleotides (3-6). miRNAs regulate gene expression via binding the 3 '-untranslated region (3'-UTR) of the targeted mRNAs to suppress the translation or induce the degradation of messenger RNAs (mRNAs) (5). It has been well-documented that miRNAs play important roles in a variety of physiological conditions, including cell growth, differentiation and apoptosis (7). The oncogenic or tumor-suppressive function of miRNAs is involved in the initiation and progression of human cancers $(8,9)$. Notably, increasing evidence has demonstrated that miRNAs play important roles in regulating the proliferation, invasion and metastasis of NSCLC $(10,11)$. For example, highly expressed miR-10 was found to promote the growth of NSCLC cells via targeting phosphatase and tensin homolog (PTEN) (12). miR-17 was found to regulate cisplatin-resistance and metastasis of NSCLC (13). A recent study showed that miR-187-3p mitigated the proliferation of NSCLC cells by modulating the expression of BCL6 (14). Collectively, these studies indicate that miRNAs are important regulators which modulate the growth of NSCLC cells.

miR-221-3p is a newly identified oncogenic miRNA involved in the development of tumors $(15,16)$. A recent study showed that miR-221-3p is upregulated in pancreatic cancer and serves as a potential biomarker for the diagnosis of cancer patients (16). miR-221-3p was also found to promote the metastasis of cervical cancer via targeting the Twist homolog 2 (THBS2) (15). Additionally, the oncogenic function of miR-221-3p has also been identified in gastric cancer, and enhanced the growth of gastric cancer cells by inhibiting the expression of PTEN (17). Interestingly, downregulation of miR-221-3p has been suggested as a prognostic biomarker in triple-negative breast cancer (TNBC) and was found to be associated with the poor prognosis of TNBC patients (18). The oncogenic or tumor-suppressive function of miR-221-3p may depend on the tumor system. Recent research has proposed miR-221-3p as a possible therapeutic tool (19). These findings indicate the critical involvement of miR-221-3p in cancers; 
however, the involvement of miR-221-3p in NSCLC has not been well investigated.

The present study aimed to evaluate the expression and function of miR-221-3p in NSCLC. The results uncovered that miR-221-3p was highly expressed in NSCLC tissues and cell lines. Overexpression of miR-221-3p promoted the growth of NSCLC cells via targeting the cell cycle regulator p27 and enhanced cell cycle progression.

\section{Materials and methods}

Cell lines and tissue samples. The NSCLC cell lines A549, H1299, H23 and SK-MES-1, and the normal bronchial epithelium BEAS-2B were purchased from the American Type Culture Collection (ATCC; Manassas, VA, USA). The cells were cultured in Dulbecco's modified Eagle's medium $\left(\mathrm{Gibco}^{\mathrm{TM}}\right.$ DMEM; Thermo Fisher Scientific, Inc., Waltham, MA, USA) supplemented with $10 \%$ fetal bovine serum (FBS; Thermo Fisher Scientific, Inc.), $100 \mathrm{U} / \mathrm{ml}$ of penicillin and $100 \mu \mathrm{g} / \mathrm{ml}$ of streptomycin (Invitrogen; Thermo Fisher Scientific, Inc.) at $37^{\circ} \mathrm{C}$ with $5 \% \mathrm{CO}_{2}$. For cell transfection, $20 \mathrm{nM}$ control miRNA (5'-CGG UACGAUCGCGGCGGGAUAUC-3') or 20 nM miR-221-3p (5'-AGCUACAUUGUCUGCUGGGUUUC-3') was transfected into $1 \times 10^{4}$ NSCLC cells using Lipofectamine 2000 reagent (Invitrogen; Thermo Fisher Scientific, Inc.) according to the manufacturer's instructions. The cells were harvested for the following experiments after transfection for $48 \mathrm{~h}$.

The 50-paired NSCLC tissues and matched adjacent normal lung tissues were collected from patients (age range, 35-75 years; female to male ratio, 1:0.78) who were diagnosed at Longnan Hospital (Daqing, Heilongjiang, China) between April 2011 and August 2013. None of these patients received radiotherapy or chemotherapy prior to surgical resection. All samples were confirmed by histopathological examination and stored in liquid nitrogen before use. Written informed consent was obtained from all the participants. The sample collection was approved by the Institutional Review Board of Longnan Hospital (Daqing, Heilongjiang, China). The experiments using these samples were performed according to the Declaration of Helsinki.

RNA extraction and quantitative PCR. Total miRNA was extracted from the tissues using the miRcute miRNA isolation kit (Tiangen, Beijing, China) according to the manufacturer's instructions. Reverse transcription (RT) PCR was performed using the One Step Prime Script miRNA cDNA Synthesis kit (Takara, Dalian, China). The quantitative PCR (qPCR) was conducted with TaqMan ${ }^{\mathrm{TM}}$ Multiplex Master Mix (Invitrogen; Thermo Fisher Scientific, Inc.) using the Applied Biosystems 7500 Real-Time RT-PCR system (Thermo Fisher Scientific, Inc.). The expression of U6 RNA was detected as the normalization control. The primers for miR-221-3p and U6 were designed as follows: miR-221-3p forward, 5'-ACACTCCAGCTGGGA GCTACA and reverse, 5'-CTCAACTGGTGTCGTGGA; U6 forward, 5'-ATTGGAACGATACAGAG and reverse, 5'-GGA ACGCTTCACGAATTT. The thermocycling conditions were as follows: Initial denaturation at $95^{\circ} \mathrm{C}$ for $5 \mathrm{~min}$ followed by 40 cycles at $95^{\circ} \mathrm{C}$ for $10 \mathrm{sec}$ and $60^{\circ} \mathrm{C}$ for $1 \mathrm{~min}$. The relative expression of miR-221-3p was calculated with the $2^{-\Delta \Delta C q}$ method (20). The experiments were performed in triplicate.
Cell proliferation. Both A549 and H1299 cells were seeded into a 96 -well plate at a density of $2 \times 10^{3}$ cells per well. The cell proliferation was quantified by adding $10 \mu \mathrm{l}$ of Cell Counting Kit- 8 reagent (CCK-8, Dojindo, Kumamoto, Japan) and incubated at $37^{\circ} \mathrm{C}$ for $2 \mathrm{~h}$. The absorbance of each well at $450 \mathrm{~nm}$ was measured with a microplate reader.

Cell cycle analysis. Cells transfected with the corresponding miRNAs were cultured in 60-mm dishes. When the cell confluence reached approximately $70 \%$, the cells were harvested and fixed with pre-cold $70 \%$ ethanol overnight at $4^{\circ} \mathrm{C}$. The cells were washed twice with PBS and incubated with $1 \mathrm{mg} / \mathrm{ml}$ RNase A at $37^{\circ} \mathrm{C}$ for $30 \mathrm{~min}$. Afterwards, cells were stained with $50 \mu \mathrm{g} / \mathrm{ml}$ propidium iodide (PI) for $40 \mathrm{~min}$ at $37^{\circ} \mathrm{C}$. Cells were then passed through a 300-mesh nylon net and the cell cycle distribution was determined by flow cytometry (Becton Dickinson FACS Calibur; BD Biosciences, Franklin Lakes, NJ, USA).

Wound-healing assay. NSCLC cells were cultured in 6-well plates and transfected with the indicated miRNA. After transfection, the cells were incubated for $36 \mathrm{~h}$, and the wound was generated using a $200 \mu \mathrm{l}$ pipette tip. Cells were washed twice with PBS buffer. The wound region was detected after 24 h by light microscopy (magnification, $\mathrm{x} 40$ ).

Cell invasion. The cell invasion assay was performed using 24-well polycarbonate Transwell chambers with $8-\mu \mathrm{m}$ pores (Corning, Inc.). In total, $1 \times 10^{3} \mathrm{NSCLC}$ cells/well transfected with the control miRNA or miR-221-3p were plated in serum-free DMEM in the upper chamber. Inserts were precoated with Matrigel. DMEM with $10 \%$ FBS was added to the lower chambers. After incubation for $36 \mathrm{~h}$ at $37^{\circ} \mathrm{C}$, invaded cells were stained with $0.1 \%$ crystal violet for $10 \mathrm{~min}$ at room temperature and quantified. Cells were visualized and counted using a light microscope (magnification, x40).

Dual-luciferase reporter assay. The 3'-UTR of p27 containing the targeting sites of miR-221-3p was amplified and cloned into the p-MIR-reporter plasmid (Ambion; ThermoFisherScientific, Inc.). NSCLC cells cotransfected with miR-221-3p and the luciferase reporter vector of $\mathrm{p} 27$ were plated $\left(1 \times 10^{3}\right.$ cells/well) in 96-well plates. The Renilla luciferase vector was also transfected with Lipofectamine 2000 (Invitrogen; Thermo Fisher Scientific, Inc.) as control of the transfection efficiency. After transfection for $48 \mathrm{~h}$, the luciferase activity was measured with the Dual-Luciferase Reporter Assay System (Promega Corporation) according to the manufacturer's protocol. The p-MIR-firefly (Ambion; Thermo Fisher Scientific, Inc.) luciferase activity was normalized to p-MIR-Renilla (Ambion; Thermo Fisher Scientific, Inc.) activity.

Bioinformatics prediction. The databases of TargetScan (http://www.Targetscan.org) and miRBase (http://www. mirbase.org) were used to predict the potential targets of miR-221-3p by inputting the name of miRNA in the query.

Western blot analysis. After transfection for $48 \mathrm{~h}$, cells were harvested and lysed with the NP-40 buffer [150 mM $\mathrm{NaCl}, 1 \% \mathrm{NP}-40,50 \mathrm{mM}$ Tris- $\mathrm{HCl}$ (pH 8.0), 1 mM EDTA] 

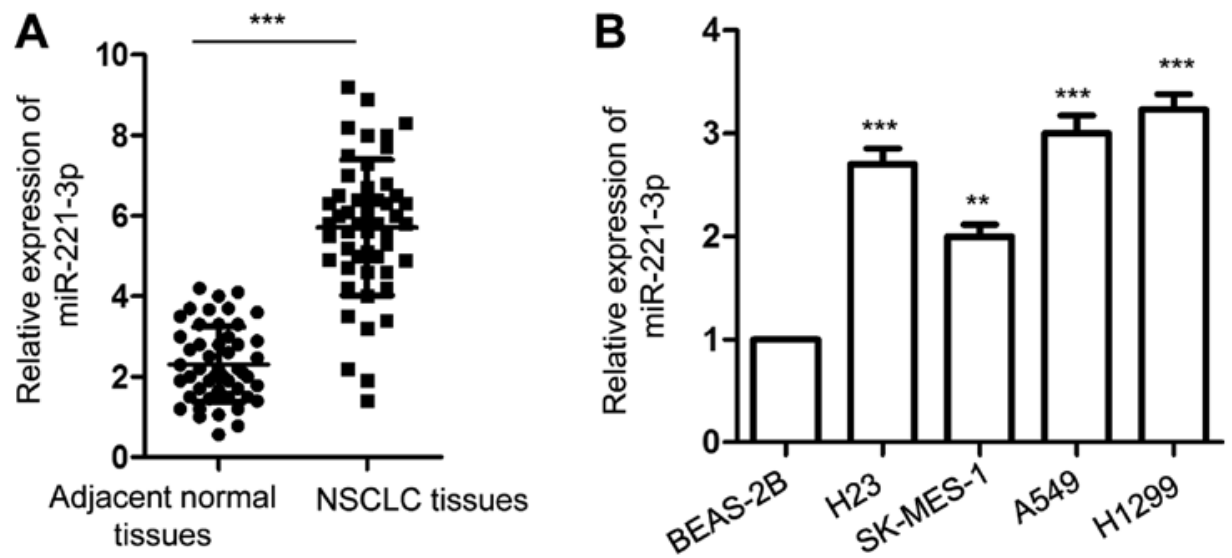

Figure 1. miR-221-3p is overexpressed in NSCLC. (A) The expression of miR-221-3p in paired NSCLC and adjacent normal tissues was detected by RT-qPCR. (B) Expression of miR-221-3p in NSCLC cell lines (H23, SK-MES-1, A549 and H1299) and normal bronchial epithelium BEAS-2B cells was compared with RT-qPCR assay. ${ }^{* *} \mathrm{P}<0.01$ and ${ }^{* * * *} \mathrm{P}<0.001$ vs. corresponding control. NSCLC, non-small cell lung cancer; RT-qPCR, reverse transcription-quantitative PCR.

containing $0.15 \mathrm{U} / \mathrm{ml}$ aprotinin, $20 \mathrm{mM}$ leupeptin and $1 \mathrm{mM}$ phenylmethylsulfonyl fluoride. Proteins were loaded onto the $15 \%$ SDS-PAGE and transferred onto nitrocellulose filter membranes (Pall Life Sciences, Port Washington, NY, USA). The membrane were initially blocked with $5 \%$ non-fat milk for $1 \mathrm{~h}$ at room temperature (RT) and then incubated with the primary antibody overnight at $4^{\circ} \mathrm{C}$. The membranes were then incubated with the secondary antibody for $1 \mathrm{~h}$ at RT. The western blot bands were visualized with the Amersham $^{\mathrm{TM}}$ ECL Plus Western Blotting Detection System (GE Healthcare, UK). The antibodies used in this study included anti-p27 (cat. no. sc-1641, Santa Cruz Biotechnology, Inc., Dallas, TX, USA; dilution ratio: 1:2,000), anti-GAPDH (cat. no. 3H12, MBL, Japan; dilution ratio: 1:3,000) and anti-Flag (cat. no. ab1257; Abcam, Cambridge, MA, USA; dilution ratio: $1: 2,000)$ which were purchased from the mentioned companies. The intensities of the protein bands were analyzed using the Image J software (version D1.47; National Institutes of Health).

Cell apoptosis analysis. The percentage of cell apoptosis was assessed using PI/Annexin V-based flow cytometry with the Annexin V-FITC Apoptosis Detection kit (Thermo Fisher Scientific, USA) according to the manufacturer's instructions. Briefly, cells were harvested and washed with pre-cold PBS. Cells were re-centrifuged and resuspended to a final density of $\sim 1 \times 10^{6}$ cells $/ \mathrm{ml}$ with the Annexin-binding buffer. $5 \mu \mathrm{l}$ of FITC/Annexin V and $1 \mu \mathrm{l}$ of $100 \mu \mathrm{g} / \mathrm{ml} \mathrm{PI}$ working solution was added to each $100 \mu \mathrm{l}$ of cell suspension. After incubation for $15 \mathrm{~min}$ at $\mathrm{RT}, 400 \mu \mathrm{l}$ of $1 \mathrm{X}$ Annexin-binding buffer was added into the cells and mixed gently. The cell apoptosis was analyzed by flow cytometry as soon as possible.

Statistical analysis. Data are presented as mean \pm standard deviation (SD). Statistical analysis was examined with SPSS 19.0 software version (IBM Corp., Armonk, NY, USA). Student's t-test was used to analyze the difference between two groups. One-way analysis of variance followed by Dunnett's test was adopted when comparing more than two groups. $\mathrm{P}<0.05$ was considered to be statistically significant.

\section{Results}

miR-221-3p is overexpressed in NSCLC tissues and cell lines. To investigate the involvement of miR-221-3p in NSCLC, the expression of miR-221-3p in 50-paired NSCLC tissues and matched corresponding normal lung tissues was detected with RT-qPCR. The data showed that the expression of miR-221-3p was significantly increased in NSCLC tissues compared with that in the adjacent normal tissues (Fig. 1A). Additionally, the abundance of miR-221-3p in NSCLC cell lines including A549, H1299, H23 and SK-MES-1 and normal bronchial epithelium BEAS-2B cells were also investigated. As presented in Fig. 1B, a significantly higher level of miR-221-3p was obtained in the NSCLC cell lines than that noted in the normal cells. These results indicated the overexpression of miR-221-3p in NSCLC.

Downregulation of miR-221-3p suppresses the growth of NSCLC cells. As the expression of miR-221-3p was overexpressed in NSCLC, we investigated the influence of miR-221-3p on the growth of NSCLC cells. Thus, miR-221-3p was downregulated by transfecting miR-221-3p antagomir into A549 and H1299 cells. The downregulation efficiency of miR-221-3p was validated by RT-qPCR analysis (Fig. 2A). The effect of miR-221-3p on the proliferation of NSCLC cells was detected with the CCK-8 assay. As presented in Fig. 2B and C, suppression of miR-221-3p significantly inhibited the proliferation of both A5459 and H1299 cell lines in comparison with the cells transfecting with the negative control miRNA. To further confirm these results, an in vitro wound-healing assay was performed with A549 and H1299 cells expressing downregulated miR-221-3p. Decreased expression of miR-221-3p markedly reduced the migration of both A549 and H1299 cell lines (Fig. 2D). Furthermore, the effect of miR-221-3p downregulation by antagomir transfection on the apoptosis of NSCLC cells was also investigated. As shown in Fig. 2E, depletion of miR-221-3p significantly increased the relative cell apoptosis percentage of both A549 and H1299 cell lines. The effect of miR-221-3p on the invasion of NSCLC cells was also detected by transfecting negative control miRNA or miR-221-3p antagomir into A549 and H1299 cells. The result showed that downregulation of miR-221-3p significantly 
A

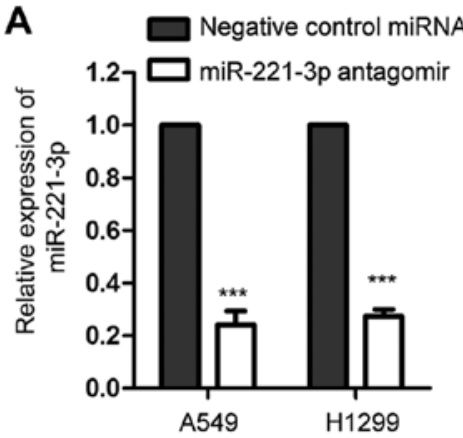

D

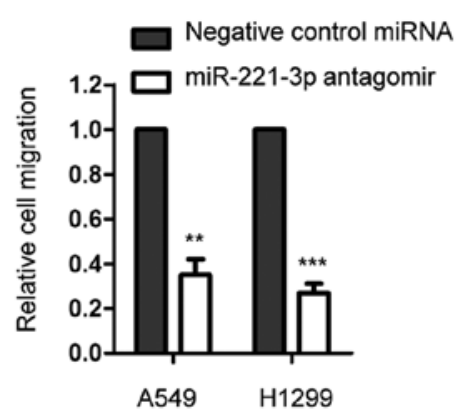

B

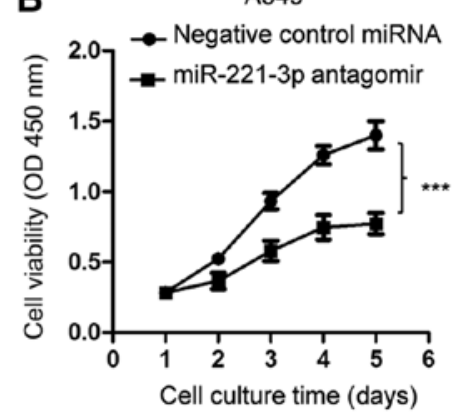

C

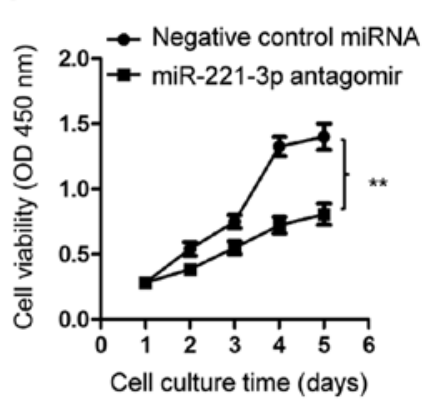

E
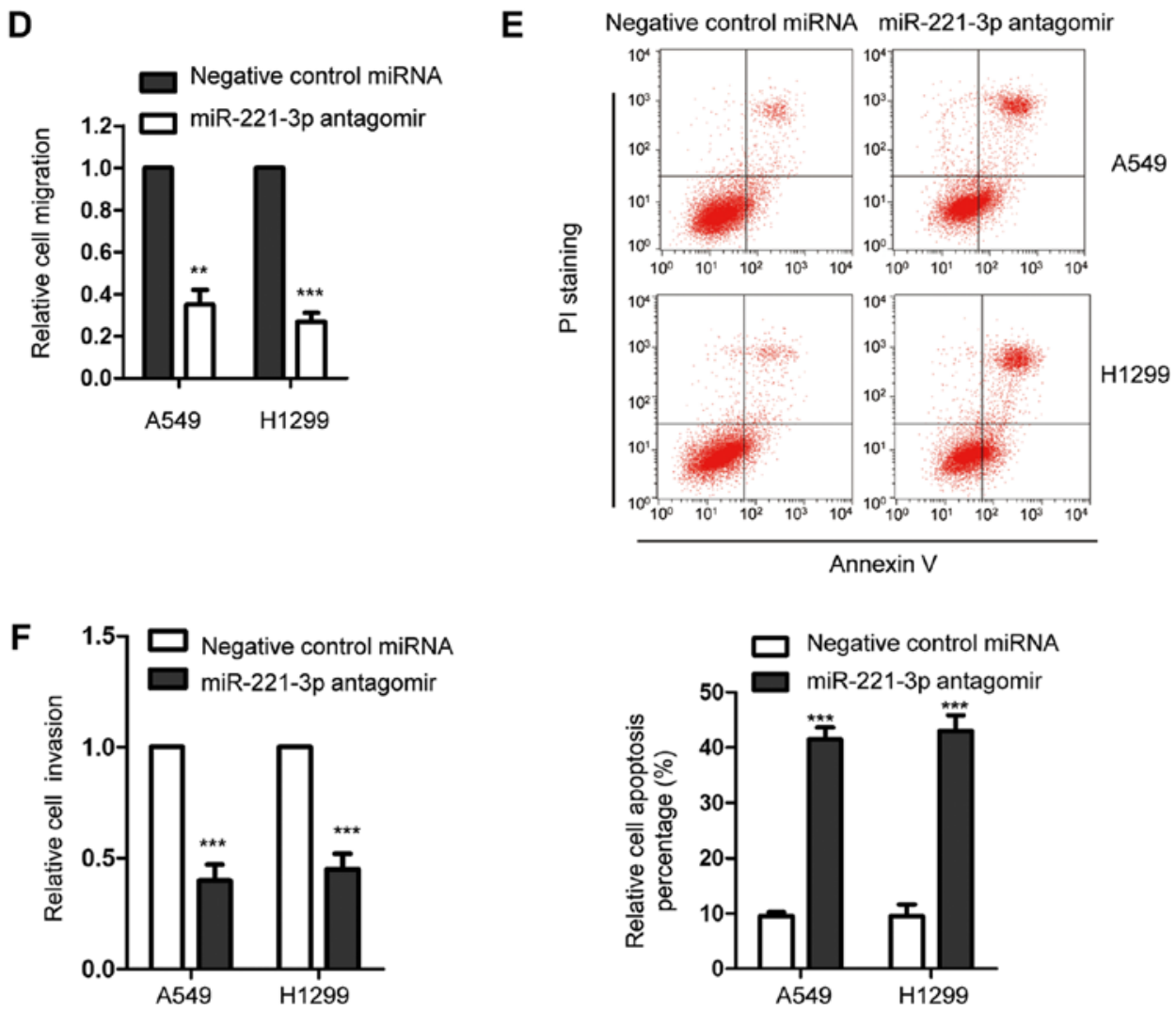

Figure 2. Downregulation of miR-221-3p inhibits the growth of NSCLC cells. (A) Expression of miR-221-3p in the A549 and H1299 cell lines transfected with miR-221-3p antagomir or negative control miRNA. (B and C) The proliferation of A549 and H1299 cells transfected with control miRNA or miR-221-3p antagomir was detected with the CCK-8 assay. (D) Depletion of miR-221-3p inhibited the migration of NSCLC cells. (E) Effect of miR-221-3p downregulation on the apoptosis of both A549 and H1299 cells by flow cytometry. (F) Knockdown of miR-221-3p suppressed the invasive ability both A549 and H1299 cell lines. ${ }^{* *} \mathrm{P}<0.01$ and ${ }^{* * *} \mathrm{P}<0.001$ vs. corresponding control. NSCLC, non-small cell lung cancer.

inhibited the invasion of NSCLC cells (Fig. 2F). Collectively, these data demonstrated that depletion of miR-221-3p suppressed the growth of NSCLC cells.

p27 is a target of miR-221-3p in NSCLC cells. The prediction of RNA-associated interactions with computational prediction algorithms plays important roles in characterizing the function of miRNAs (21). To further understand the molecular mechanisms by which miR-221-3p regulates the growth of NSCLC cells, the potential targets of miR-221-3p were predicted with the bioinformatics resource TargetScan (http://www.Targetscan.org) and miRBase (http://www. mirbase.org) (Tables SI and SII). The data showed that p27, the inhibitor of cell cycle progression, which plays important roles in the initiation and progression of cancers $(22,23)$, ranked highly among all the possible targets. The putative binding sites of miR-221-3p at the 3'-UTR of p27 are presented as Fig. 3A. To detect the binding between miR-221-3p with the 3'-UTR of p27, luciferase assay was performed by transfecting luciferase reporter plasmids containing wild-type (WT) or mutant 3'-UTR of p27 in the presence of miR-221-3p mimics in A549 and H1299 cells. The results showed that the luciferase activity of WT but not the mutant 3'-UTR of p27 was significantly decreased with the co-transfection of miR-221-3p mimics (Fig. 3B and C). This result suggested the binding between miR-221-3p and the 3'-UTR of p27. To detect the consequence of the binding, the mRNA and protein levels of p27 in the NSCLC cell lines with overexpression of miR-221-3p were examined, respectively. As presented in Fig. 3D, high expression of miR-221-3p significantly decreased the mRNA level of p27. Consistently, the protein level of p27 was also inhibited upon the overexpression of miR-221-3p in 
B

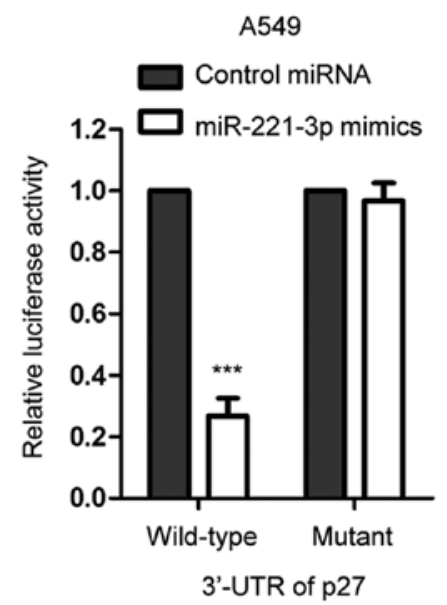

C

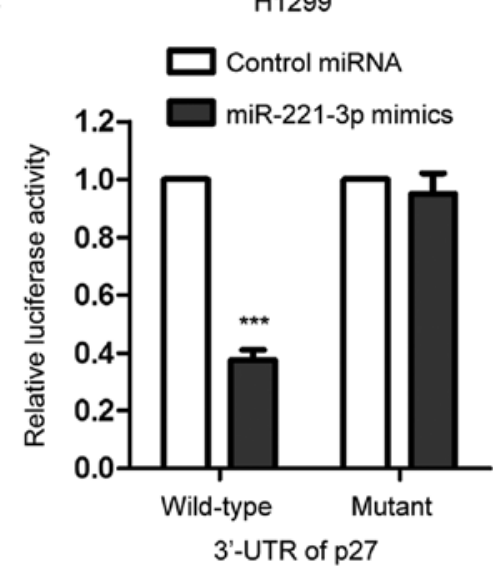

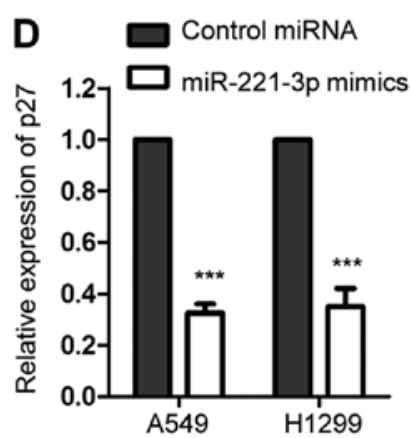
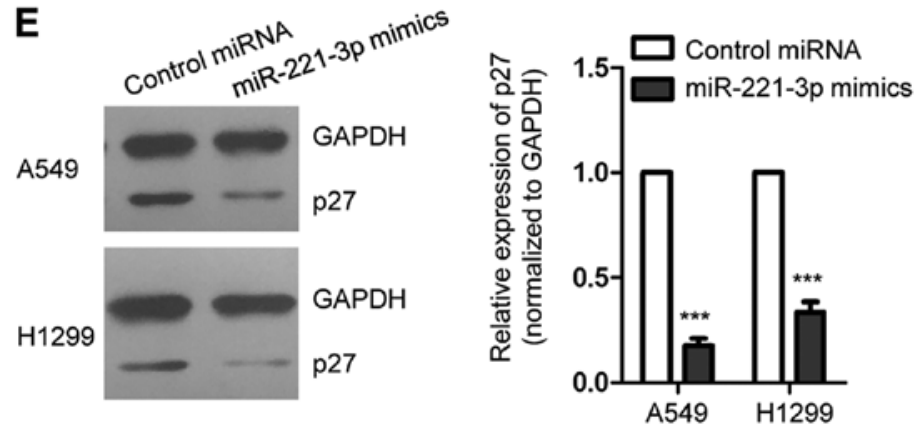

Figure 3. miR-221-3p targets p27 in NSCLC cells. (A) Binding sites of miR-221-3p and p27. (B and C) Overexpression of miR-221-3p decreased the luciferase activity of wild-type (WT) p27 3'-UTR in A549 and H1299 cells. (D) The mRNA level of p27 was detected by RT-qPCR in the A549 and H1299 cells with the transfection of control miRNA or miR-221-3p antagomir. (E) Downregulation of miR-221-3p significantly decreased the expression of p27 in NSCLC cells. ${ }^{* * *} \mathrm{P}<0.001$ vs. control miRNA. NSCLC, non-small cell lung cancer; UTR, untranslated region; RT-qPCR, reverse transcription-quantitative PCR.

the A549 and H1299 cell lines (Fig. 3E). These results indicated that miR-221-3p was able to bind the 3'-UTR of p27 and suppress the expression of p27 in NSCLC cells.

Overexpression of miR-221-3p promotes the cell cycle progression of NSCLC cells. An increased level of p27 has been reported to control the cell cycle progression at G1 phase (24). As miR-221-3p was found to negatively regulate the expression of $\mathrm{p} 27$, to ascertain the influence of miR-221-3p on the cell cycle progression of NSCLC cells, both A549 and H1299 cell lines were transfected with miR-221-3p mimics or control miRNA, and the cell cycle progression was assessed by flow cytometry. As presented in Fig. 4A and B, compared with the control cells, overexpression of miR-221-3p significantly promoted the cell cycle progression from $\mathrm{G} 1$ to $\mathrm{S}$ phase in the A549 and H1299 cell lines. This result was consistent with the decreased expression of p27 with the ectopic expression of miR-221-3p in NSCLC cells. To further confirm this observation, endogenous miR-221-3p was downregulated by inducing miR-221-3p antagomir into the NSCLC cells. RT-qPCR and western blot analysis were performed to detect the expression level of p27. The data showed that depletion of miR-221-3p increased the abundance of p27 in the
A549 and H1299 cell lines (Fig. 4C and D). The cell cycle progression of NSCLC cells expressing negative control miRNA or miR-221-3p antagomir was examined by flow cytometry. As shown in Fig. 4E and F, downregulation of miR-221-3p significantly induced cell cycle arrest at the G1 phase. These results collectively indicated that miR-221-3p is a novel regulator of the cell cycle progression of NSCLC cells. To detect whether the modulation of miR-221-3p on the proliferation of NSCLC cells was by regulating p27, both A549 and H1299 cell lines were transfected with Flag empty vector or Flag-p27 (Fig. 4G). The CCK-8 assay indicated that overexpression of miR-221-3p promoted the proliferation of the NSCLC cell lines, and transfection of p27 significantly decreased the growth of NSCLC cells expressing miR-221-3p (Fig. 4H and I). These results suggest that p27 mediates the role of miR-221-3p in NSCLC.

\section{Discussion}

Non-small cell lung cancer (NSCLC) is a heavy burden for human health worldwide. Exploring novel factors involved in the progression of NSCLC will benefit the prognosis and treatment of NSCLC patients. In the present study, we 
A

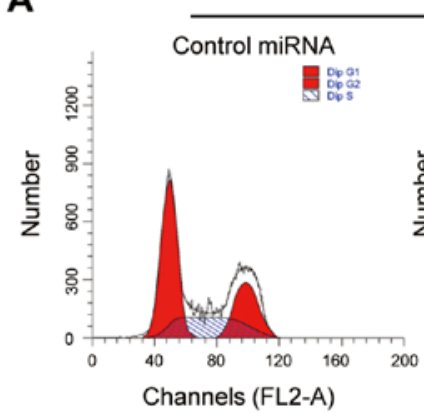

\section{A549}

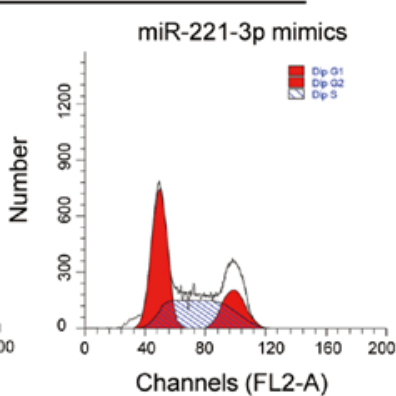

B
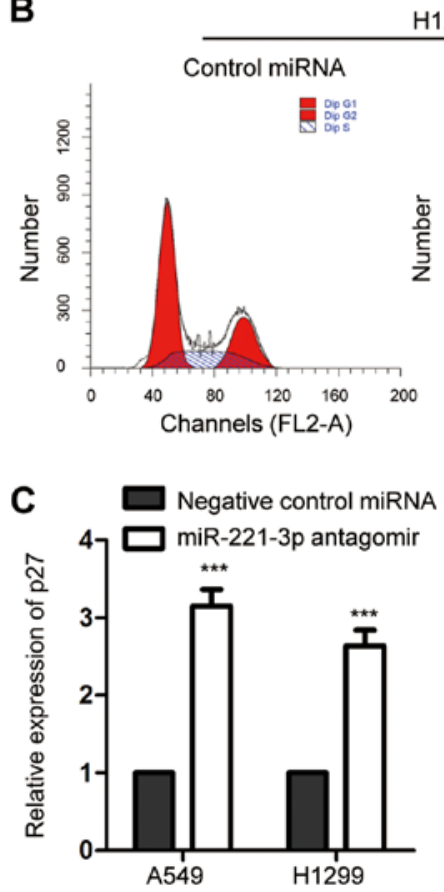

H1299

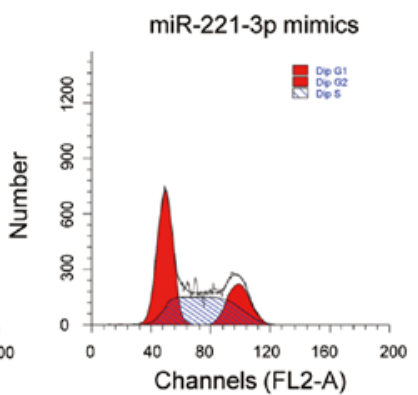

D $\begin{array}{cc}\text { Negative control miR-221-3p } \\ \text { miRNA } & \text { antagomir }\end{array}$

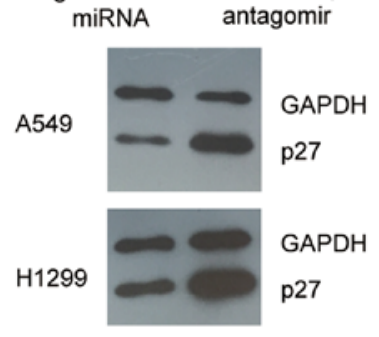

549

E

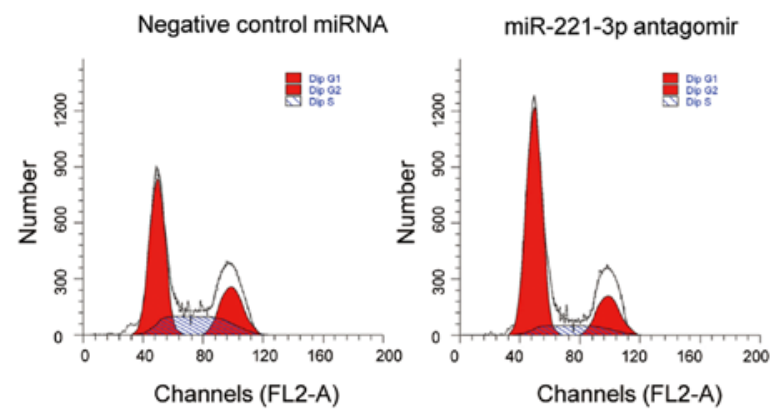

F

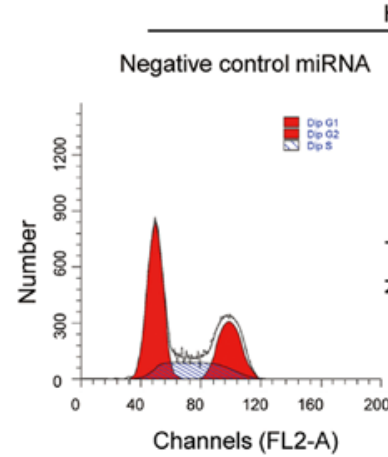

$\mathrm{H} 1299$

miR-221-3p antagomir
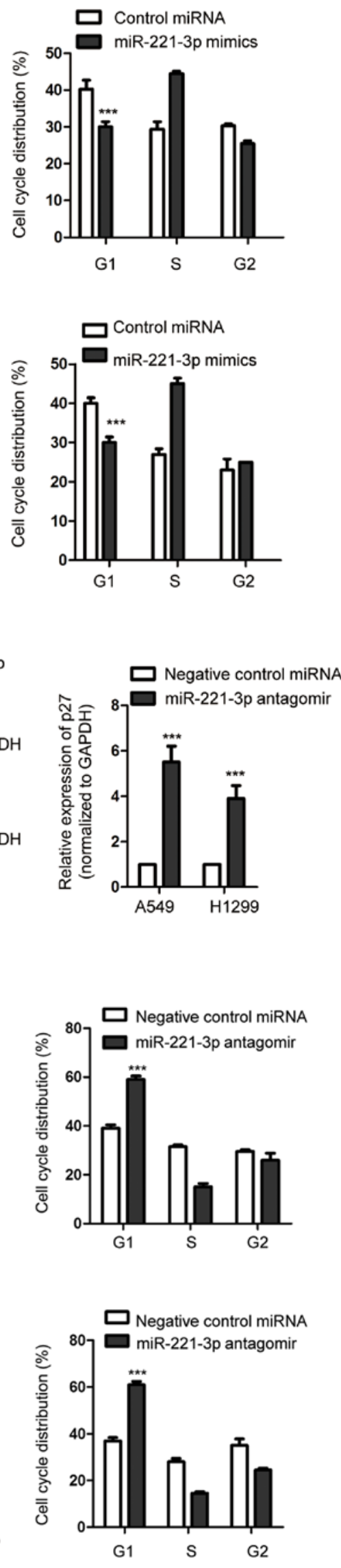

Figure 4. Overexpression of miR-221-3p promotes the cell cycle progression of NSCLC cells. (A and B) A549 and H1299 cells were transfected with control miRNA or miR-221-3p mimics and the cell cycle distribution was examined by flow cytometry. (C and D) Downregulation of miR-221-3p significantly decreased the mRNA and protein expression of p27. (E and F) Suppression of miR-221-3p expression induced cell cycle arrest at the G1 phase of NSCLC cells. ${ }^{* * *} \mathrm{P}<0.001$ vs. corresponding control. NSCLC, non-small cell lung cancer. 

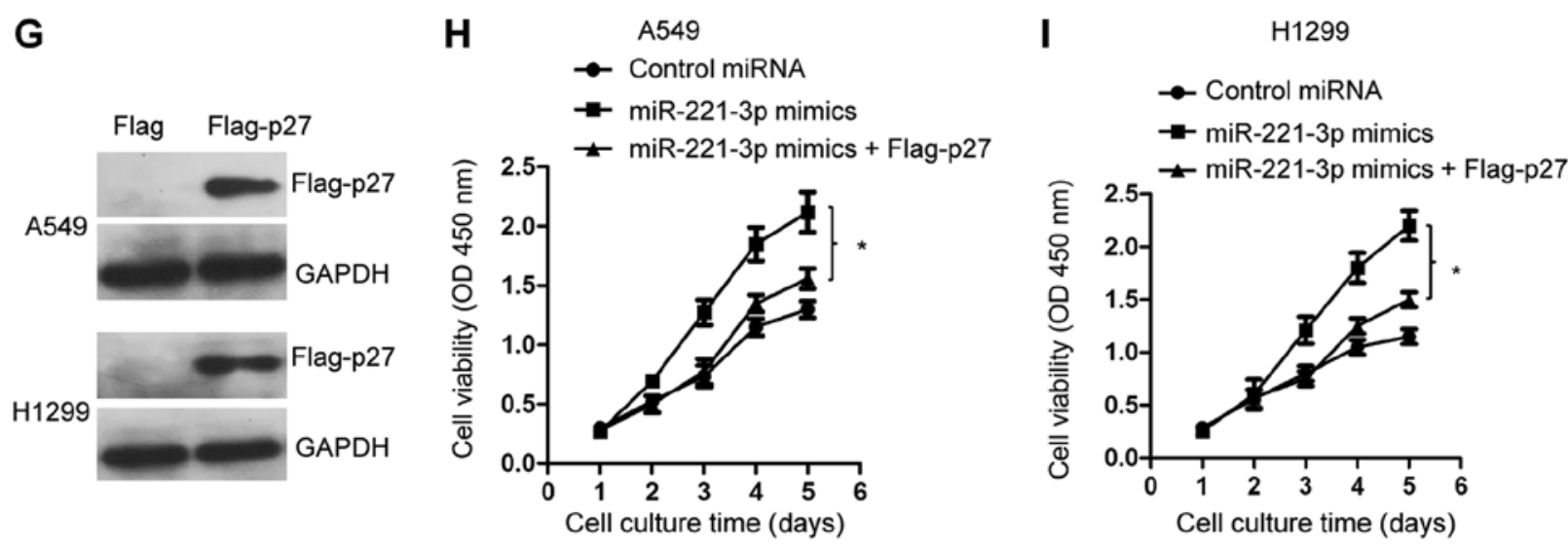

Figure 4. Continued. Overexpression of miR-221-3p promotes the cell cycle progression of NSCLC cells. (G) Both A549 and H1299 cells were transfected with Flag vector or Flag-p27, and the overexpression of p27 was confirmed with anti-Flag antibody. (H and I) Overexpression of p27 reversed the positive effect of miR-221-3p on the proliferation of NSCLC cells. ${ }^{*} \mathrm{P}<0.05$ and ${ }^{* * *} \mathrm{P}<0.001$ vs. corresponding control. NSCLC, non-small cell lung cancer.

evaluated the expression of miR-221-3p in NSCLC tissues and cell lines. High expression of miR-221-3p was observed in NSCLC tissues compared with the normal control tissues. Further mechanistic study found that overexpression of miR-221-3p suppressed the growth of NSCLC cells via targeting p27.

Consistent with the high expression of miR-221-3p in NSCLC tissues, a previous study showed that overexpression of miR-221-3p maintained tumor necrosis factor (TNF)-related apoptosis-inducing ligand (TRAIL) in NSCLC, which indicated that miR-221-3p is a possible target for the diagnosis of TRAIL resistance in NSCLC (25). A recent study demonstrated that miR-221-3p is overexpressed in pancreatic cancer and serves as a potential biomarker for the diagnosis of cancer patients (16). In addition, the oncogenic function of miR-221-3p was also identified in gastric cancer, which targeted PTEN and enhanced the growth of gastric cancer cells (17). It was also reported that cervical squamous cell carcinoma-secreted exosomal miR-221-3p promoted the lymphangiogenesis and lymphatic metastasis (26). miR-221-3p was upregulated by the transcription factor TWIST2 and targeted THBS2 in cervical cancer, which promoted lymph node metastasis (15). Different from the potential oncogenic role of miR-221-3p in the above reports, a recent study by $\mathrm{Wu}$ et al (27) found that a higher level of miR-221-3p in epithelial ovarian cancer (EOC) inhibited the growth of cells and was associated with the better overall survival of EOC patients, which indicated the tumor-suppressive role of miR-221-3p in EOC. Consistently, a high level of miR-221-3p was associated with better 5-year disease-free survival of the triple-negative breast cancer patients (18). Combined with these findings, the regulation of miR-221-3p on cell growth might be associated with cancer types. In the present study, downregulation of miR-221-3p increased the apoptosis of NSCLC cells. It may be interesting to further analyze this miR-221-3p-associated cell death with the bioinformatics resource such as ncRDeathDB (28).

The cyclin-dependent kinase inhibitor p27 is a cell cycle regulator belonging to the Cip/Kip family that inhibits the cell cycle progression at the G1/S boundary $(29,30)$. A low level of p27 was found to be correlated with rapid proliferation in a variety of cancer types (31). The abundance of $\mathrm{p} 27$ expression is regulated by diverse mechanisms including transcription, translation and protein degradation (32). Additionally, multiple oncogene-activated pathways target p27 to trigger its cytoplasmic translocation, which consequently attenuates the nuclear cell cycle inhibitory functions and allows activation of CDK1 and CDK2 (33). Emerging evidence suggests that miRNAs control the cell cycle progression of cancer cells by modulating the expression of p27 (34). For example, miR-199a-5p was found to target p27 in osteosarcoma and to contribute to cancer cell growth (35). Recent results by Wang et al (36) demonstrated that miR-200c directly regulates the expression of p27 and enhances the proliferation of gastric cancer cells. p27 has also been identified as the target of miR-222 in hepatocellular carcinoma (HCC) to regulate the proliferation of HCC cells (37). Downregulation of miR-221-3p inhibited the proliferation of pancreatic cancer cells via upregulating the expression of $\mathrm{p} 27$ (38). In the present study, it was found that miR-221-3p is an upstream regulator of p27 in NSCLC cells. miR-221-3p was able to bind the 3'-UTR of p27 and inhibit the expression of p27 in NSCLC cells. Consistent with the decreased level of p27 with miR-221-3p, overexpression of miR-221-3p significantly promoted the cell cycle progression of NSCLC cells. Due to the inhibition of p27 expression upon the overexpression of miR-221-3p, the correlation between the expression of miR-221-3p with the clinical parameters of NSCLC is a significant issue which warrants further study. Additionally, the subcellular location of miR-221-3p deserves further investigation with the available software as the location might affect the function and structure of miRNAs (39). The involvement of miR-221-3p in other types of cancer or diseases can be predicted with the bioinformatic databases $(40,41)$.

In summary, our findings found that miR-221-3p was highly expressed in NSCLC tissues and cell lines. Molecular mechanistic studies revealed that miR-221-3p targets p27 and promotes the cell cycle progression of NSCLC cells. Our results uncovered the possible mechanisms of miR-221-3p in NSCLC, which indicate that miR-221-3p might be considered as a promising anticancer target in NSCLC. 


\section{Acknowledgements}

Not applicable.

\section{Funding}

No funding was received.

\section{Availability of data and materials}

The datasets used and/or analyzed during the current study are available from the corresponding author on reasonable request.

\section{Authors' contributions}

GY and JL designed the study, performed the experiments and analyzed the data. BZ collected the tissue samples and performed some experiments. JL wrote the manuscript.

\section{Ethics approval and consent to participate}

Written informed consent was obtained from all the participants. The sample collection was approved by the Institutional Review Board of Longnan Hospital (Daqing, Heilongjiang, China). The experiments using these samples were performed according to the Declaration of Helsinki.

\section{Patient consent for publication}

Not applicable.

\section{Competing interests}

The authors declare that they have no competing interests.

\section{References}

1. Vestergaard HH, Christensen MR and Lassen UN: A systematic review of targeted agents for non-small cell lung cancer. Acta Oncol 57: 176-186, 2018.

2. Xiong Y, Wang T, Wang M, Zhao J, Li X, Zhang Z, Zhou Y, Liu J, Jia $L$ and Han Y: Long non-coding RNAs function as nove predictors and targets of non-small cell lung cancer: A systematic review and meta-analysis. Oncotarget 9: 11377-11386, 2018.

3. Ambros V: The functions of animal microRNAs. Nature 431 350-355, 2004

4. Bartel DP: MicroRNAs: Genomics, biogenesis, mechanism, and function. Cell 116: 281-297, 2004.

5. Fabian MR, Sonenberg N and Filipowicz W: Regulation of mRNA translation and stability by microRNAs. Annu Rev Biochem 79: 351-379, 2010.

6. Mohr AM and Mott JL: Overview of microRNA biology. Semin Liver Dis 35: 3-11, 2015.

7. Kwak PB, Iwasaki S and Tomari Y: The microRNA pathway and cancer. Cancer Sci 101: 2309-2315, 2010.

8. Farazi TA, Spitzer JI, Morozov P and Tuschl T: miRNAs in human cancer. J Pathol 223: 102-115, 2011.

9. Gentilin E, Degli Uberti E and Zatelli MC: Strategies to use microRNAs as therapeutic targets. Best Pract Res Clin Endocrinol Metab 30: 629-639, 2016.

10. Ma R, Wang C, Wang J, Wang D and Xu J: miRNA-mRNA interaction network in non-small-cell lung cancer. Interdiscip Sci 8 : 209-219, 2016.

11. Zhang W, Zhang Q, Zhang M, Zhang Y, Li F and Lei P: Analysis for the mechanism between the small cell lung cancer and non-small cell lung cancer combing the miRNA and mRNA expression profiles. Thorac Cancer 6: 70-79, 2015.
12. Yu T, Liu L, Li J, Yan M, Lin H, Liu Y, Chu D, Tu H, Gu A and Yao M: MiRNA-10a is upregulated in NSCLC and may promote cancer by targeting PTEN. Oncotarget 6: 30239-30250, 2015.

13. Jiang Z, Yin J, Fu W, Mo Y, Pan Y, Dai L, Huang H, Li S and Zhao J: MiRNA 17 family regulates cisplatin-resistant and metastasis by targeting TGFbetaR2 in NSCLC. PLoS One 9: e94639, 2014

14. Sun C, Li S, Yang C, Xi Y, Wang L, Zhang F and Li D: MicroRNA-187-3p mitigates non-small cell lung cancer (NSCLC) development through down-regulation of BCL6. Biochem Biophys Res Commun 471: 82-88, 2016.

15. Wei WF, Zhou CF, Wu XG, He LN, Wu LF, Chen XJ, Yan RM, Zhong M, Yu YH, Liang L and Wang W: MicroRNA-221-3p, a TWIST2 target, promotes cervical cancer metastasis by directly targeting THBS2. Cell Death Dis 8: 3220, 2017.

16. Li F, Xu JW, Wang L, Liu H, Yan Y and Hu SY: MicroRNA-221-3p is up-regulated and serves as a potential biomarker in pancreatic cancer. Artif Cells Nanomed Biotechnol 46: 482-487, 2018.

17. Shi J, Zhang Y, Jin N, Li Y, Wu S and Xu L: MicroRNA-221-3p plays an oncogenic role in gastric carcinoma by inhibiting PTEN expression. Oncol Res 25: 523-536, 2017.

18. Deng L, Lei Q, Wang Y, Wang Z, Xie G, Zhong X, Wang Y, Chen N, Qiu Y, Pu T, et al: Downregulation of miR-221-3p and upregulation of its target gene PARP1 are prognostic biomarkers for triple negative breast cancer patients and associated with poor prognosis. Oncotarget 8: 108712-108725, 2017.

19. Garofalo M, Quintavalle C, Romano G, Croce CM and Condorelli G: miR221/222 in cancer: Their role in tumor progression and response to therapy. Curr Mol Med 12: 27-33, 2012.

20. Livak KJ and Schmittgen TD: Analysis of relative gene expression data using real-time quantitative PCR and the 2(-Delta Delta C(T)) method. Methods 25: 402-408, 2001.

21. Yi Y, Zhao Y, Li C, Zhang L, Huang H, Li Y, Liu L, Hou P, Cui T, Tan P, et al: RAID v2.0: An updated resource of RNA-associated interactions across organisms. Nucleic Acids Res 45: D115-D118, 2017.

22. Raghu D, Paul PJ, Gulati T, Deb S, Khoo C, Russo A, Gallo E, Blandino G, Chan AL, Takano E, et al: E6AP promotes prostate cancer by reducing p27 expression. Oncotarget 8: 42939-42948, 2017.

23. Vasile Bochis O, Achimas-Cadariu P, Vlad C, Fetica B, Corneliu Leucuta D, Ioan Busuioc C and Irimie A: The prognostic role of Skp2 and the tumor suppressor protein p27 in colorectal cancer. J BUON 22: 1122-1130, 2017.

24. Ventura C, Nunez M, Gaido V, Pontillo C, Miret N, Randi A and Cocca $\mathrm{C}$ : Hexachlorobenzene alters cell cycle by regulating p27-cyclin E-CDK2 and c-Src-p27 protein complexes. Toxicol Lett 270: 72-79, 2017.

25. Garofalo M, Quintavalle C, Di Leva G, Zanca C, Romano G, Taccioli C, Liu CG, Croce CM and Condorelli G: MicroRNA signatures of TRAIL resistance in human non-small cell lung cancer. Oncogene 27: 3845-3855, 2008.

26. Zhou CF, Ma J, Huang L, Yi HY, Zhang YM, Wu XG, Yan RM, Liang L, Zhong M, Yu YH, et al: Cervical squamous cell carcinoma-secreted exosomal miR-221-3p promotes lymphangiogenesis and lymphatic metastasis by targeting VASH1. Oncogene 38: 1256-1268, 2019.

27. Wu Q, Ren X, Zhang Y, Fu X, Li Y, Peng Y, Xiao Q, Li T, Ouyang C, Hu Y, et al: MiR-221-3p targets ARF4 and inhibits the proliferation and migration of epithelial ovarian cancer cells. Biochem Biophys Res Commun 497: 1162-1170, 2018.

28. Wu D, Huang Y, Kang J, Li K, Bi X, Zhang T, Jin N, Hu Y, Tan P, Zhang L, et al: ncRDeathDB: A comprehensive bioinformatics resource for deciphering network organization of the ncRNA-mediated cell death system. Autophagy 11: 1917-1926, 2015.

29. Sgambato A, Cittadini A, Faraglia B and Weinstein IB: Multiple functions of p27(Kip1) and its alterations in tumor cells: A review. J Cell Physiol 183: 18-27, 2000.

30. Guan X, Wang Y, Xie R, Chen L, Bai J, Lu J and Kuo MT: p27(Kipl) as a prognostic factor in breast cancer: A systematic review and meta-analysis. J Cell Mol Med 14: 944-953, 2010.

31. Borriello A, Bencivenga D, Criscuolo M, Caldarelli I, Cucciolla V, Tramontano A, Borgia A, Spina A, Oliva A, Naviglio S and Della Ragione F: Targeting p27Kip1 protein: Its relevance in the therapy of human cancer. Expert Opin Ther Targets 15: 677-693, 2011.

32. Wander SA, Zhao D and Slingerland JM: p27: A barometer of signaling deregulation and potential predictor of response to targeted therapies. Clin Cancer Res 17: 12-18, 2011. 
33. Sharma SS and Pledger WJ: The non-canonical functions of p27(Kip1) in normal and tumor biology. Cell Cycle 15: 1189-1201, 2016.

34. Fernandez S, Risolino M and Verde P: A novel miRNA-mediated STOP sign in lung cancer: miR-340 inhibits the proliferation of lung cancer cells through p27(KIP1). Mol Cell Oncol 2: e977147, 2015.

35. Wang C, Ba X, Guo Y, Sun D, Jiang H, Li W, Huang Z, Zhou G, Wu S, Zhang J and Chen J: MicroRNA-199a-5p promotes tumour growth by dual-targeting PIAS3 and p27 in human osteosarcoma. Sci Rep 7: 41456, 2017.

36. Wang Y, Zeng J, Pan J, Geng X, Liu Y, Wu J, Song P, Wang Y, Jia J and Wang L: MicroRNA-200c is involved in proliferation of gastric cancer by directly repressing p27(Kip1). Biochem Biophys Rep 8: 227-233, 2016.

37. Yang YF, Wang F, Xiao JJ, Song Y, Zhao YY, Cao Y, Bei YH and Yang CQ: MiR-222 overexpression promotes proliferation of human hepatocellular carcinoma HepG2 cells by downregulating p27. Int J Clin Exp Med 7: 893-902, 2014

38. Sarkar S, Dubaybo H, Ali S, Goncalves P, Kollepara SL, Sethi S, Philip PA and Li Y: Down-regulation of miR-221 inhibits proliferation of pancreatic cancer cells through up-regulation of PTEN, p27(kip1), p57(kip2), and PUMA. Am J Cancer Res 3: 465-477, 2013.
39. Zhang T, Tan P, Wang L, Jin N, Li Y, Zhang L, Yang H, Hu Z, Zhang L, Hu C, et al: RNALocate: A resource for RNA subcellular localizations. Nucleic Acids Res 45: D135-D138, 2017.

40. Cui T, Zhang L, Huang Y, Yi Y, Tan P, Zhao Y, Hu Y, Xu L, Li E and Wang D: MNDR v2.0: An updated resource of ncRNA-disease associations in mammals. Nucleic Acids Res 46: D371-D374, 2018.

41. Li Y, Wang C, Miao Z, Bi X, Wu D, Jin N, Wang L, Wu H, Qian K, Li C, et al: ViRBase: A resource for virus-host ncRNA-associated interactions. Nucleic Acids Res 43: D578-D582, 2015.

This work is licensed under a Creative Commons Attribution-NonCommercial-NoDerivatives 4.0 International (CC BY-NC-ND 4.0) License. 